\title{
Response to Dr. Costellos' submission entitled "Re: Vitamin and mineral use and risk of prostate cancer: the case-control surveillance: Zhang et al. Cancer Causes Control. 2008 Dec 18. [Epub ahead of print]"
}

\author{
Yuqing Zhang $\cdot$ Patricia F. Coogan • \\ Brian L. Strom · Lynn Rosenberg
}

Received: 9 March 2009/Accepted: 1 April 2009/Published online: 16 April 2009

(C) Springer Science+Business Media B.V. 2009

We agree with Drs. Franklin and Costello that the study of weak associations in observational epidemiologic studies is difficult. In a well-conducted randomized trial of zinc supplement use, in relation to incidence of prostate cancer, differences between the treated and untreated groups that could bias the results would be small or absent, if the study was large enough. In the absence of such a randomized trial, however, there is no alternative to reliance on the weight of evidence from observational epidemiologic studies and other relevant (e.g., laboratory) investigations. Despite some inconsistencies, which are usually the situation for weak associations, the weight of evidence from epidemiologic studies at present is in the direction of an

\section{Y. Zhang $(\bowtie)$}

Clinical Epidemiology Research and Training Unit, Boston

University School of Medicine, Boston, MA, USA

e-mail: yuqing@bu.edu

\section{P. F. Coogan · L. Rosenberg}

Slone Epidemiology Center, Boston University, Boston,

MA, USA

B. L. Strom

Center for Clinical Epidemiology and Biostatistics, University

of Pennsylvania School of Medicine, Philadelphia, PA, USA

B. L. Strom

Department of Biostatistics and Epidemiology, University

of Pennsylvania School of Medicine, Philadelphia, PA, USA

B. L. Strom

Center for Education and Research on Therapeutics, University

of Pennsylvania School of Medicine, Philadelphia, PA, USA

B. L. Strom

Division of General Internal Medicine of the Department of Medicine, University of Pennsylvania School of Medicine, Philadelphia, PA, USA adverse effect. Our results add to that evidence. We make no claim for having established cause and effect. In addition, we agree with the limitations of our study that Drs. Franklin and Costello had pointed out; having ourselves pointed them out in our paper. We had noted that we had no information on dosage of the supplements. We also had commented that since zinc has been linked to prostate health, some men with early symptoms of cancer might have self-medicated with zinc supplements, which could have contributed to the positive findings. Let us hope that evidence will be forthcoming from well-conducted randomized trials. In their absence, however, we must make judgments based on the best evidence available, even if it is imperfect. 